\title{
The Role of Eddy Diffusivity Profiles on Stratocumulus Liquid Water Path Biases
}

\author{
STEPHAN R. DE ROODE \\ Royal Netherlands Meteorological Institute (KNMI), De Bilt, Netherlands
}

(Manuscript received 5 July 2006, in final form 20 October 2006)

\begin{abstract}
Results from simulations of the stratocumulus-topped boundary layer with one-dimensional versions of general simulation models typically exhibit a wide range of spread in the modeled liquid water path (LWP). These discrepancies are often attributed to differences in the modeled entrainment rate. Results from a large eddy simulation of the First International Satellite Cloud Climatology Project Regional Experiment I stratocumulus case are analyzed. The diagnosed eddy diffusivities for heat and moisture are found to differ by about a factor of 3. Moreover, both have a much larger magnitude than the ones typically applied in boundary layer parameterization schemes. Motivated by these results mean state solutions are analyzed for the specific case in which the vertical fluxes of heat and moisture are prescribed, whereas eddy diffusivity profiles are systematically varied by multiplication with a constant factor. The solutions demonstrate that any value, ranging from zero to a maximum adiabatic value, can be obtained for the LWP. In the subtropical parts over the ocean where horizontally extended stratocumulus fields persist, the surface sensible heat flux is typically small, whereas surface evaporation and entrainment of relatively dry air from above the surface can result in significant moisture fluxes. If the eddy diffusivity values are small, then the mean specific humidity will tend to decrease quite rapidly with height in order to support the humidity flux. This results in erroneous low humidity values in the upper part of the boundary layers causing low LWP values.
\end{abstract}

\section{Introduction}

The representation of stratocumulus cloud layers in general circulation models (GCMs) is problematic. For example, Duynkerke and Teixeira (2001) compared results from the European Centre for Medium-Range Weather Forecasts (ECMWF) Re-Analysis (ERA) results for July 1987 with stratocumulus observations for the same period collected during the First International Satellite Climatology Project (ISCCP) Regional Experiment (FIRE). They concluded that in ERA the cloud cover and liquid water path (LWP) are strongly underestimated. These results inspired a recent model intercomparison study in which one-dimensional versions of GCMs, or so-called single column models (SCMs), were used to simulate the diurnal cycle of stratocumulus off the coast of California as observed dur-

Corresponding author address: Stephan R. de Roode, Royal Netherlands Meteorological Institute (KNMI), P. O. Box 201, 3730 AE De Bilt, Netherlands.

E-mail: roode@knmi.nl

DOI: 10.1175/MWR3426.1

(C) 2007 American Meteorological Society ing FIRE I (Duynkerke et al. 2004). Although the initial thermodynamic state, the sea surface temperature, and the large-scale subsidence rate were prescribed, the cloud LWP evolution differed considerably among the SCMs. This discrepancy was attributed predominantly to differences in the entrainment velocity, which is the rate with which relatively warm and dry air from just above the inversion is mixed into the cloud-topped boundary layer.

Analyses of aircraft measurements indicate that the entrainment velocity has typical values in the range between 2 and $20 \mathrm{~mm} \mathrm{~s}^{-1}$ (Nicholls and Turton 1986; De Roode and Duynkerke 1997; Faloona et al. 2005). A difficulty with prognosing the stratocumulus evolution is that even small differences in the entrainment rate, say on the order of just a few millimeters per second, can lead to significant differences in the LWP. For example, Stevens (2002) showed that different entrainment parameterizations yielded solutions for the LWP that differed almost by a factor of 3 .

However, Stevens et al. (2005) conclude that differences in the entrainment rates cannot fully explain the 
variability found in modeled LWP results. They analyzed results from several large eddy simulation (LES) models that participated in an intercomparison study of a stratocumulus case based on observations collected during the Second Dynamics and Chemistry of Marine Stratocumulus field study (DYCOMS-II). Whereas the simulated LWPs differed more than a factor of 5, differences in the LWP of perfect mixed layers constructed using the mean thermodynamic properties of the simulations would only differ by a factor of 2 . Simulations that produced the smallest LWPs had larger gradients in moisture within the boundary layer. In other words, the redistribution of moisture within the boundary layer by turbulent transport is a vital factor controlling the LWP. A large spread in the LWP was also found from various SCM simulations of the same DYCOMS-II stratocumulus case (Zhu et al. 2005). These differences were attributed to the sensitivity of liquid water content to small changes in total humidity and temperature induced by the different turbulent transport and microphysics scheme employed.

In a GCM, the vertical turbulent transport $\overline{w^{\prime} \psi^{\prime}}$ of a scalar $\psi$ can be computed with an eddy diffusivity approach,

$$
\overline{w^{\prime} \psi^{\prime}}(z)=-K_{\psi}(z) \frac{\partial \bar{\psi}(z)}{\partial z},
$$

with $z$ denoting the height. The flux is thus taken proportionally to the product of an eddy diffusivity $K_{\psi}$ and the vertical gradient of the grid-box mean value of the scalar. The closure problem is then reduced to finding suitable functions for $K_{\psi}$, where it should be noted that eddy diffusivities for momentum and thermodynamic quantities usually differ, hence, $K_{\psi}$ is scalar dependent. Since $K_{\psi}$ has dimensions of meters squared per second it scales with the product of a turbulent velocity scale and an appropriate length scale $l$ characterizing the dominant eddy size. In a (turbulent kinetic energy) TKE-l scheme the eddy diffusivity is computed as

$$
K_{\psi}=\sqrt{E} l .
$$

This approach requires an additional equation to solve the TKE $(E)$. Various length scale formulations for a TKE- $l$ scheme are proposed by Bougeault and Lacarrère (1989), Lenderink and Holtslag (2004), and Sánchez and Cuxart (2004). Troen and Mahrt (1986) and Louis (1979) present eddy diffusivity parameterizations that do not use the TKE but consider the local stability of the boundary layer.

According to Stull (1988), "there has been no lack of creativity by investigators in designing parameteriza- tions for $K_{\psi}$. " The question whether this actually matters is the major topic of this note. In particular, solutions for the LWP are analyzed for different eddy diffusivity profiles. We neglect precipitation and eliminate effects due to differences in entrainment rates by using fixed, prescribed vertical flux profiles for heat and moisture.

\section{Modeling results of eddy diffusivities for the EUROCS stratocumulus intercomparison case}

\section{a. Large eddy simulation results}

A large eddy simulation was performed as part of the European Project on Cloud Systems in Climate Models (EUROCS) stratocumulus intercomparison study, which was designed on the basis of observations collected in stratocumulus off the coast of California during FIRE I (Duynkerke et al. 2004). The Dutch Atmospheric LES (DALES) model used a large domain $\left(25.6 \times 25.6 \times 1.2 \mathrm{~km}^{3}\right)$ with $256 \times 256 \times 80$ grid points.

Figure 1 shows examples of vertical profiles of the horizontal slab-mean values of the total specific humidity $q_{t}$ and the liquid water potential temperature $\theta_{l}$ and their respective vertical turbulent fluxes, $\overline{w^{\prime} q_{t}^{\prime}}$ and $\overline{w^{\prime} \theta_{l}^{\prime}}$. Both quantities are conserved variables for moistadiabatic processes, $\theta_{l}=\theta-\left(L_{v} / c_{p} \pi\right) q_{l}$, where $\theta$ is the potential temperature, $q_{l}$ is the liquid water content, $L_{v}$ is the latent heat of vaporization of water, and $c_{p}$ is the specific heat at constant pressure of dry air. The Exner function $\pi$ gives the ratio of absolute to potential temperature. The jumps in the mean $\theta_{l}$ and $q_{t}$ clearly mark the inversion layer. The sharp jump in $\overline{w^{\prime} \theta_{l}^{\prime}}$ results from a strong longwave radiative cooling near the top of the cloud layer.

Figure 2 shows an example of eddy diffusivity profiles $K_{\theta_{l}}$ and $K_{q_{i}}$. These results were diagnosed with aid of Eq. (1) using the results shown in Fig. 1. It is clear that the eddy diffusivities for heat and moisture are different. In the lower half of the boundary layer $K_{\theta_{l}}$ can be well approximated by $K_{q_{t}}$ multiplied by a factor of 0.35 .

Wyngaard and Brost (1984) and De Roode et al. (2004) presented different vertical profiles for the eddy diffusivities for bottom-up $\left(s_{\mathrm{bu}}\right)$ and top-down $\left(s_{\mathrm{td}}\right)$ scalars in the clear convective boundary layer and the stratocumulus-topped boundary layer, respectively. A bottom-up scalar has a surface flux $\left|\overline{w^{\prime} s_{\mathrm{bu}, 0}^{\prime}}\right|>0$ and a zero flux at the top of the boundary layer due to entrainment, $\overline{w^{\prime} s_{\mathrm{bu}, T}^{\prime}}=0$, and vice versa for the top-down scalar. The application of the principle of linear superposition of variables enables to reconstruct any arbitrary eddy diffusivity $K_{\psi}$, provided that the scalar $\psi$ is a con- 

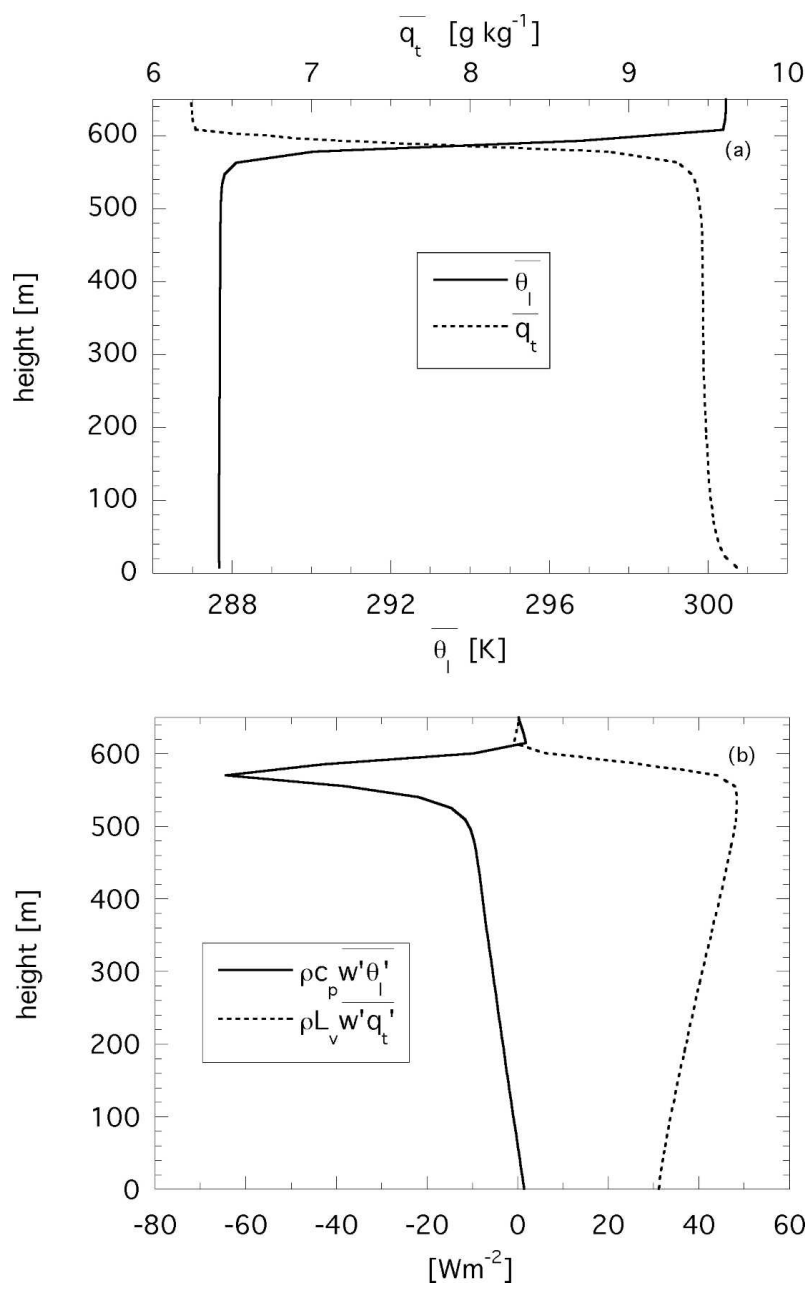

FIG. 1. Vertical profiles of the (a) mean and (b) vertical fluxes of the liquid water potential temperature $\theta_{l}$ and the total specific humidity $q_{t}$. The results represent horizontal slab-mean values computed during the third hour of an LES of the FIRE-I stratocumulus case. Line styles are explained in the legend.

served variable. To this end, let us consider the tendency equation for the mean,

$$
\frac{\partial \bar{\psi}}{\partial t}=-\frac{\overline{w^{\prime} \psi^{\prime}}}{\partial z}+S_{\psi}
$$

where we have collected terms like the mean advection, radiation, precipitation, etc., into a single source term $S_{\psi}$. If we set the source term to zero, the above tendency equation is linear in $\psi$. This means that if at $t=0$ $\psi$ can be expressed as a linear superposition of the bottom-up and top-down scalar fields, this will also be the case for any $t>0$ (Jonker et al. 1999). Let us write $\psi$ as

$$
\psi=a s_{\mathrm{bu}}+b s_{\mathrm{td}}+d,
$$

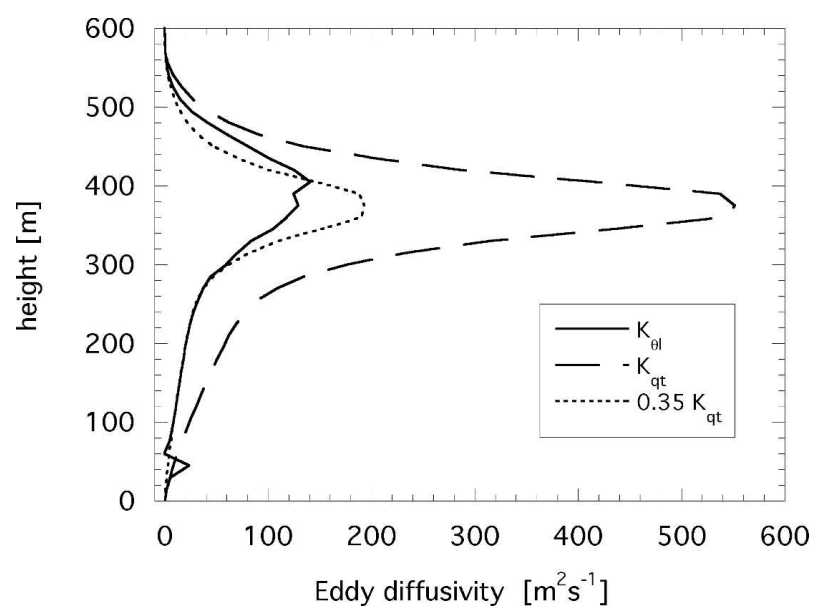

FIG. 2. Vertical profiles of the eddy diffusivity for the liquid water potential temperature $K_{\theta_{l}}$ and the total specific humidity $K_{q_{t}}$ diagnosed from the LES results shown in Fig. 1. Also shown is the $K_{q_{t}}$ profile multiplied by a factor of 0.35 . Line styles are explained in the legend.

where $d$ is an arbitrary constant. From Reynolds averaging the vertical flux $\overline{w^{\prime} \psi^{\prime}}$ can be expressed as

$$
\overline{w^{\prime} \psi^{\prime}}=a \overline{w^{\prime} s_{\mathrm{bu}}^{\prime}}+b \overline{w^{\prime} s_{\mathrm{td}}^{\prime}},
$$

with constants $a=\overline{w^{\prime} \psi_{0}^{\prime}} / \overline{w^{\prime} s_{\mathrm{bu}, 0}^{\prime}}$ and $b=\overline{w^{\prime} \psi_{T}^{\prime}} / \overline{w^{\prime} s_{\mathrm{td}, T}^{\prime}}$. Using Eq. (1), $K_{\psi}$ can be diagnosed from

$$
K_{\psi}=-\frac{\overline{w^{\prime} s_{\mathrm{bu}}^{\prime}}+\frac{b}{a} \overline{w^{\prime} s_{\mathrm{td}}^{\prime}}}{\frac{\partial \overline{s_{\mathrm{bu}}}}{\partial z}+\frac{b}{a} \frac{\partial \overline{s_{\mathrm{td}}}}{\partial z}} .
$$

The precise shape of $K_{\psi}$ will therefore generally depend on the factors $a$ and $b$, or, in other words, the entrainment to surface flux ratio of $\psi$.

Also note that the level where the $\overline{w^{\prime} \theta_{l}^{\prime}}$ flux changes sign does not depend on the cloud-top height. Because $\overline{w^{\prime} \theta_{l}^{\prime}}$ is negative at the top of the boundary layer due to entrainment of relatively warm air from just above the inversion, it will change sign if the surface sensible heat flux is positive. The zero flux level may even be located near the cloud top if the radiative cooling effect is larger than entrainment warming. Using a downgradient relation, a zero eddy diffusivity will be diagnosed at the level where the flux changes sign, provided that the mean vertical gradient has a nonzero value.

\section{b. Single-column modeling results}

To give a flavor of eddy diffusivity profiles typically applied in GCMs, results of six single-column model versions of general and regional circulation models like Action de Recherche Petite Echelle Grande Echelle 
(ARPEGE), ECMWF, the nonhydrostatic mesoscale atmospheric model of the French research community (MESO NH), Met Office (UKMO), Royal Netherlands Meteorological Institute (KNMI) Regional Atmospheric Climate Model (RACMO), and the HighResolution Limited-Area Model (HIRLAM) are presented in Fig. 3. A brief description of the diffusion schemes used by these models (except for UKMO) is given by Lenderink et al. (2004). The results represent hourly mean eddy diffusivity values used for both heat and moisture during the third hour of the simulation.

To facilitate direct comparison to the LES results, the SCMs (except for UKMO) simulated the EUROCS stratocumulus case with the precipitation scheme switched off. In addition, to focus on the performance of the eddy diffusivity scheme only the mass flux convection scheme was not used, either. Clearly, the LES and SCM results differ. For the SCMs the maximum eddy diffusivity values range from 4 to $81 \mathrm{~m}^{2} \mathrm{~s}^{-1}$. In particular the eddy diffusivities used in the SCMs for moisture are therefore much smaller than diagnosed from the LES results.

\section{Eddy diffusivity profiles and liquid water path solutions}

Mean state solutions can be diagnosed from a vertical integration of Eq. (1),

$$
\bar{\psi}(z)=\psi_{0}-\int_{0}^{z} \frac{\overline{w^{\prime} \psi^{\prime}}\left(z^{\prime}\right)}{K_{\psi}\left(z^{\prime}\right)} d z^{\prime},
$$

where $\psi_{0}$ is the surface value. Using this equation, we are interested in solutions for $\overline{\theta_{l}}$ and $\overline{q_{t}}$ as a function of the eddy diffusivity profile.

Let us consider a set of experiments with prescribed surface latent and sensible heat fluxes. Furthermore, to exclude the effect of different entrainment rates on the mean state solutions, we also prescribe the entrainment fluxes of heat and moisture at the top of the boundary layer. Last, we like to consider solutions for which the boundary layer is in a quasi-steady state:

$$
\frac{\partial}{\partial z} \frac{\partial \bar{\psi}}{\partial t}=-\frac{\partial^{2} \overline{w^{\prime} \psi^{\prime}}}{\partial z^{2}}+\frac{\partial S_{\psi}}{\partial z}=0 .
$$

Thus, for a quasi-steady state, and in case the source term $S_{\psi}=0$, the flux will vary linearly with height. However, because the LES fluxes for heat and moisture shown in Fig. 1 vary approximately linearly with height we may directly use them to solve Eq. (7) for different eddy diffusivity profiles. Note that using the LES heat flux even takes into account the effect of longwave radiative cooling at the top of the cloud layer. By using

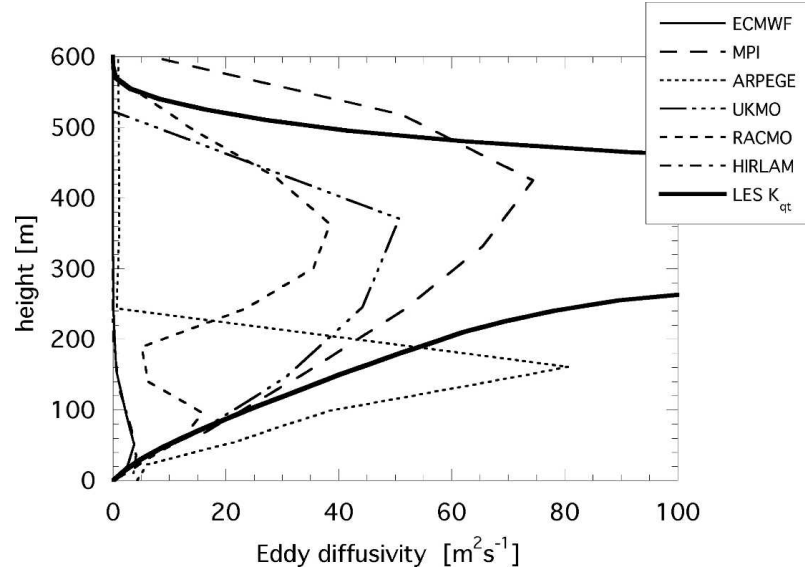

FIG. 3. Hourly-mean eddy diffusivity profiles applied to heat and moisture from six different SCM versions of general circulation models. The results were obtained from the third hour of simulations of the FIRE-I stratocumulus case. Also shown for reference is the diagnosed $K_{q_{t}}$ from the LES results presented in Fig. 2.

fixed vertical flux profiles, all experiments have the same tendencies for heat and moisture.

Different eddy diffusivity profiles $K_{c}$ are constructed from $K_{q_{t}}$ and a constant multiplication factor $c$,

$$
K_{c}=c K_{q_{t}},
$$

where the subscript $c$ denotes the value of the multiplication factor $c$. By changing the value for $c$ we can systematically vary the magnitude of $K_{c}$ while maintaining its vertical shape. We prefer to choose $K_{q_{t}}$ as a base profile because unlike $K_{\theta_{l}}$ it does not have a zero value in the lower part of the boundary layer.

Examples of vertical mean profiles are presented in Fig. 4 for $\overline{\theta_{l}}$ and $\overline{q_{t}}$. The results labeled "adiabat" indicate constant $\overline{\theta_{l}}$ and $\overline{q_{t}}$, and may also be interpreted as solutions for infinite values of the eddy diffusivity, $K_{\infty}$. In that case vertical profiles for nonconserved quantities like the temperature and liquid water content are dictated by their respective moist-adiabatic vertical gradients. Near-adiabatic stratocumulus cloud layers were frequently observed during FIRE I by Albrecht et al. (1990). The $\overline{\theta_{l}}$ solution for $c=0.35$ has a similar shape to the LES solution except near the top of the boundary layer and close to the surface where $K_{\theta_{l}}$ has a zero value. Because $K_{1}=K_{q_{t}}$ the $c=1$ solution for $\bar{q}_{t}$ is identical to the LES result depicted in Fig. 4.

The $\overline{q_{t}}$ gradients are maximum near the surface and the top of the boundary layer due to the minimum values of the eddy diffusivities at these heights. By contrast, for all $c$ values shown, the vertical profiles for $\bar{\theta}_{l}$ vary only weakly with height. With identical eddy diffusivities for heat and moisture it follows from Eq. (1) 

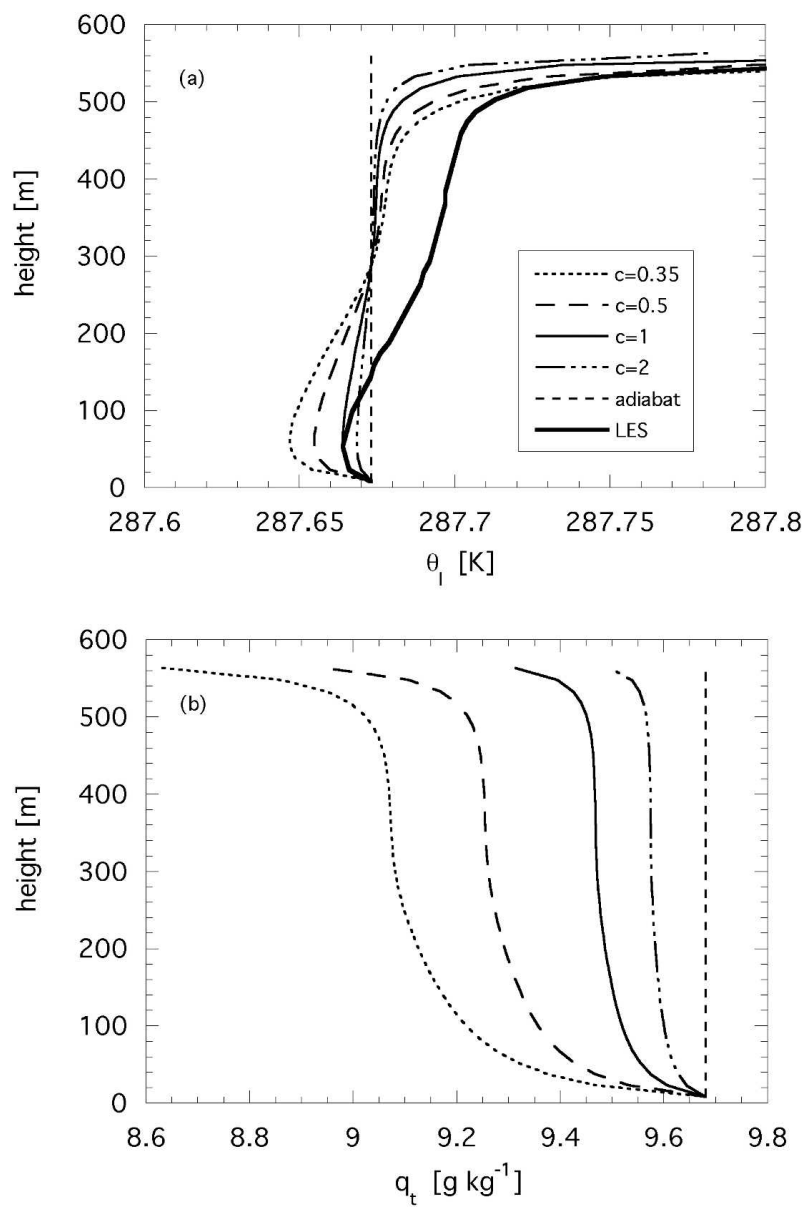

FIG. 4. Mean state solutions for (a) the liquid water potential temperature $\overline{\theta_{l}}$ and (b) the total water content $\overline{q_{t}}$. The vertical profiles were computed from Eq. (7) for the vertical flux profiles presented in Fig. 1. Different eddy diffusivity profiles were used according to Eq. (9), $K_{c}=c K_{q_{t}}$, with $K_{q_{t}}$ the eddy diffusivity diagnosed from the LES results shown in Fig. 2. The line styles are explained in the legend and represent solutions for different values for the multiplication factor $c$. The solutions labeled "adiabat" indicate constant $\theta_{l}$ and $q_{t}$ values with height. The fat gray line in (a) indicates hourly mean values from the LES model results during the third hour of simulation.

that the ratio of the mean vertical gradients of $q_{t}$ and $\theta_{l}$ can be expressed as

$$
\frac{\partial \overline{q_{t}} / \partial z}{\partial \overline{\theta_{l}} / \partial z}=\frac{\overline{w^{\prime} q_{t}^{\prime}}}{\overline{w^{\prime} \theta_{l}^{\prime}}}=\frac{c_{p} F_{q_{t}}}{L_{v} F_{\theta_{l}}},
$$

where $F_{q_{t}}$ and $F_{\theta_{l}}$ are the humidity and heat fluxes in energy units of watts per meters squared, respectively. Inserting typical fluxes observed in the subtropical parts of the ocean that are covered by stratocumulus, $F_{q_{t}}=100 \mathrm{Wm}^{-2}$ and $F_{\theta_{l}}=10 \mathrm{Wm}^{-2}$, indicates that if the change of $\bar{\theta}_{l}$ over a vertical layer with arbitrary thickness equals $0.1 \mathrm{~K}$, the corresponding change in $\overline{q_{t}}$

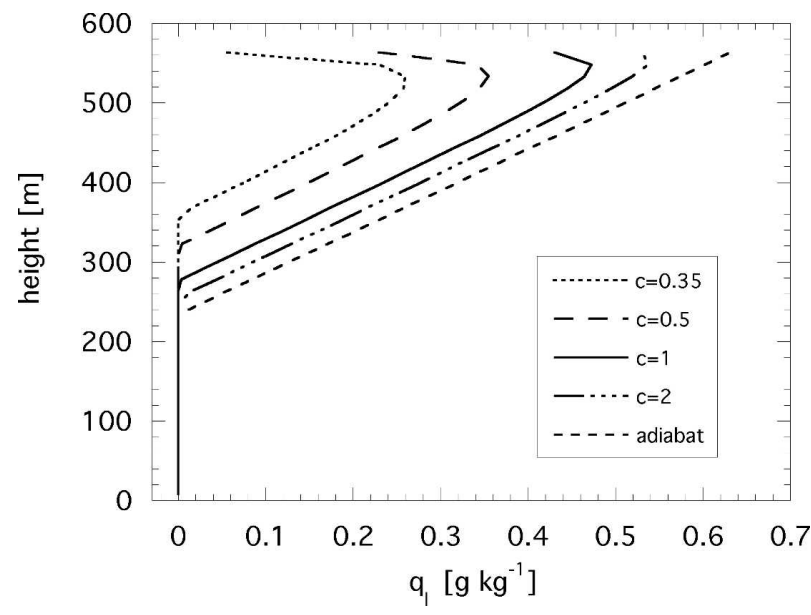

FIG. 5. The mean liquid water content $\overline{q_{l}}$ as a function of the eddy diffusivity multiplication factor $c$ according to Eq. (9). The results are diagnosed from the $\overline{\theta_{l}}$ and $\overline{q_{t}}$ solutions presented in Fig. 4. Line styles are explained in the legend.

will be $0.4 \mathrm{~g} \mathrm{~kg}^{-1}$. The relative changes are therefore much larger for $\overline{q_{t}}$ than for $\overline{\theta_{l}}$.

Also note the effect of the sign change for the vertical flux $\overline{w^{\prime} \theta_{l}^{\prime}}$ in the boundary layer. Because the flux is downgradient, a relatively large decrease in $\overline{\theta_{l}}$ below the zero-flux level will to some extent be compensated by a relatively large increase with height above. Because at all heights in the boundary layer $\overline{w^{\prime} q_{t}^{\prime}}$ is positive, $\overline{q_{t}}$ decreases monotonically with a lapse rate controlled by the multiplication factor $c$. The results illustrate that for small eddy diffusivities the total specific humidity flux can be maintained only at the expense of losing a vertically well mixed structure.

Figure 5 diagnoses the vertical profiles of the mean liquid water content $\left(\overline{q_{l}}\right)$ profile from solutions for $\overline{\theta_{l}}$ and $\overline{q_{t}}$ that were both calculated for identical $c$ values. This means that we implicitly assume that the same eddy diffusivities can be applied to $\theta_{l}$ and $q_{t}$. Although this clearly violates the results from LES, we are interested in these solutions because the SCMs discussed in this note also apply identical eddy diffusivity profiles for heat and moisture. It appears that the cloud depth and the maximum liquid water content are affected mostly by the magnitude of the mean vertical moisture gradient in the lower part of the boundary layer. Because the $q_{t}$ solution for $c=1$ equals the LES solution, and because the $\theta_{l}$ differences between the analytical profiles and LES results are rather small, the $\overline{q_{l}}$ solution for $c=1$ corresponds well to the LES results.

On the basis of vertical profiles of $\overline{q_{l}}$ we computed the LWP according to

$$
\mathrm{LWP}=\rho_{0} \int_{z_{b}}^{z_{t}} \overline{q_{l}} d z
$$




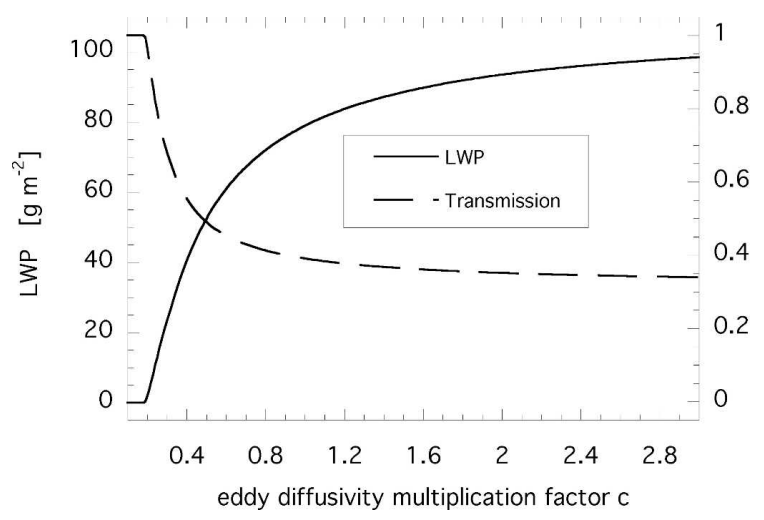

FIG. 6. LWP solutions (solid line) and the cloud transmissivity for broadband shortwave radiation (dashed line) as a function of the eddy diffusivity multiplication factor $c$ according to Eq. (9).

where the integration is performed from cloud-base to cloud-top height, and $\rho_{0}=1.14 \mathrm{~kg} \mathrm{~m}^{-3}$ is a constant reference density. It is clear from Fig. 6 that the eddy diffusivity profile has a dramatic effect on the LWP. Its values range from zero for relatively low eddy diffusivities $(c<0.19)$ to a maximum value of $109 \mathrm{~g} \mathrm{~m}^{-2}$ dictated by a moist-adiabatic stratification $(c \rightarrow \infty)$.

\section{Discussion}

In this study we analyze the mean vertical structure of a stratocumulus-topped boundary layer that is in a quasi-steady state, and for which the surface and entrainment fluxes of heat and moisture are assumed to be known. It is shown that any value for the LWP, ranging from zero to a maximum dictated by an adiabatic liquid water content, can be expected depending exclusively on the magnitudes of the eddy diffusivities used. This means that even if a model is able to accurately predict the surface and entrainment fluxes of heat and moisture, there is no guarantee that the modeled stratocumulus LWP will be in a good agreement with observations. This can be explained by the way a model distributes moisture and heat within the boundary layer. In an eddy diffusivity closure model large moisture gradients can be expected if low eddy diffusivity values are used in combination with a significant latent heat flux. A rapid decrease of the humidity with height tends to diminish the cloud LWP. It should be noted that in an LES model intercomparison study of the DYCOMS-II stratocumulus case low LWPs could also be partly explained by larger vertical total humidity gradients, in addition to different entrainment rates (Stevens et al. 2005).

The wide range of possible LWP solutions with an eddy diffusivity model may seem surprising. It gives rise to the question whether the analysis procedure uses a realistic range of eddy diffusivities? Figure 7 presents the hourly mean ECMWF eddy diffusivity profile, the humidity flux, and the (total) specific humidity profile from the ECMWF SCM simulation of the FIRE-I stratocumulus case. The maximum value for the eddy diffusivity is very small, $K=10 \mathrm{~m}^{2} \mathrm{~s}^{-1}$. Such very low eddy diffusivity values are a persistent feature in the lower part of the boundary layer in the ECMWF model simulation. Above the turbulent boundary layer a single-layer stratocumulus cloud is present. The moisture flux varies between about $20 \mathrm{~W} \mathrm{~m}^{-2}$ at the surface and $35 \mathrm{~W} \mathrm{~m}^{-2}$ due to entrainment of relatively dry air across the inversion. Clearly, the total specific humidity decreases by nearly $1.5 \mathrm{~g} \mathrm{~kg}^{-1}$ between the surface and the top of the boundary layer, in accord with the eddy diffusivity relation in (1). The low specific humidity values near the top of the boundary layer prevent the formation of clouds. In a simulation with the operational version of the ECMWF SCM, in which precipitation and convective mass flux transport were allowed, the total specific humidity at $406 \mathrm{~m}$ is only slightly larger with a value of $9.1 \mathrm{~g} \mathrm{~kg}^{-1}$.

The shape of the humidity profile shown in Fig. 7 is also remarkably similar to the mean humidity profile computed from 1 to 19 July 1987 in the paper by Duynkerke and Teixeira (2001). They conclude that the ERA model does not mix sufficiently into the cloud layer to generate a realistic stratocumulus cloud. By contrast, an analysis of the eddy diffusivity approach shows that despite the presence of significant moisture fluxes, the application of low eddy diffusivities results in low LWP values. A possible explanation for the low values for the ECMWF eddy diffusivities could be that in the subtropics the trade winds cause a mean equatorward transport of air over gradually increasing sea surface temperatures. As a result, the surface layer becomes only weakly unstable as indicated by small values for the sensible heat fluxes that are typically on the order of $10 \mathrm{~W} \mathrm{~m}^{-2}$. In the ECMWF model the eddy diffusivity in the mixed layer is proportional to a master velocity scale that depends, in turn, on the friction velocity $u_{*}$ and a convective velocity scale $w_{*}$ (Troen and Mahrt 1986). Because the latter depends on the cubic root of the surface buoyancy flux $\overline{w^{\prime} \theta_{v 0}^{\prime}}$ the convective contribution to the master velocity scale will be small.

To make the vertical profile for $\theta_{l}$ in stratocumulus more well-mixed Lock et al. (2000) suggest the inclusion of a nonlocal correction term $\gamma_{\psi}$ according to Holtslag and Boville (1993):

$$
\overline{w^{\prime} \psi^{\prime}}=-K_{\psi}\left(\frac{\partial \bar{\psi}}{\partial z}-\gamma_{\psi}\right) .
$$



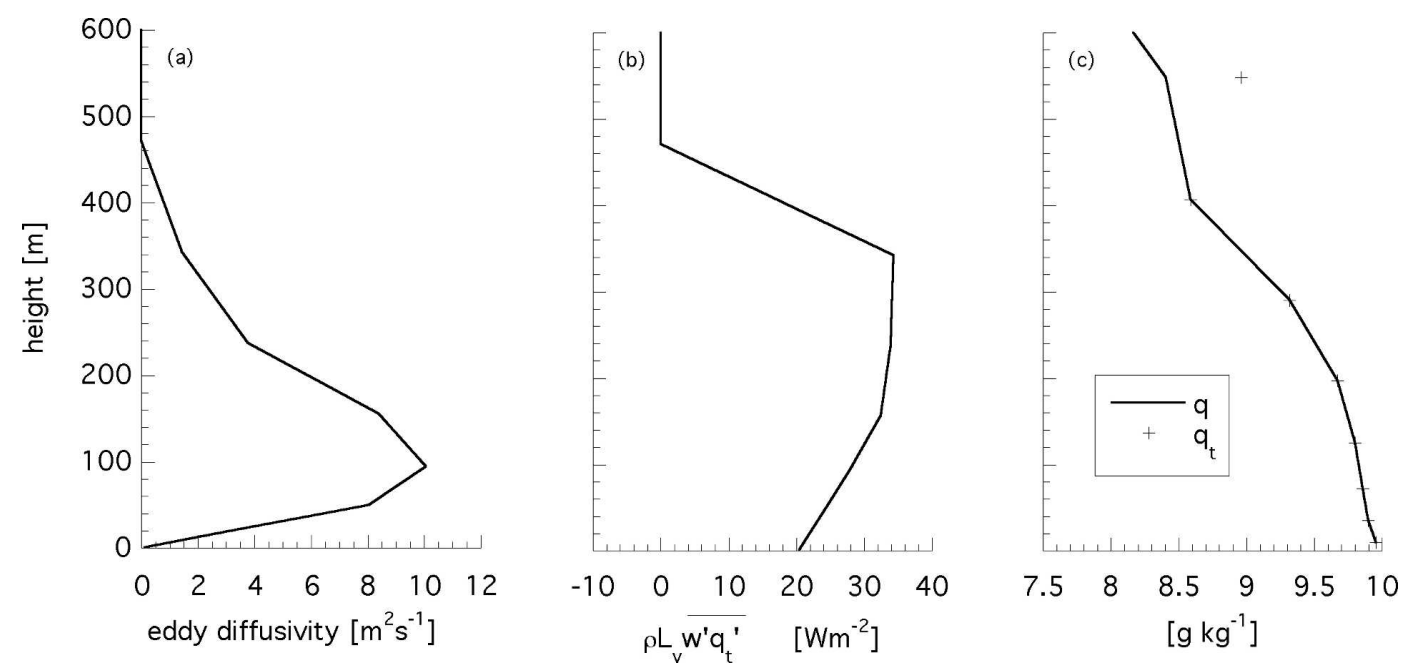

FIG. 7. Hourly-mean results from the ECMWF SCM simulation of the FIRE-I stratocumulus case. The results were computed during the 24th hour of the simulation. The eddy diffusivity applied for (a) heat and moisture, (b) the humidity flux $\rho L_{v} \overline{w^{\prime} q^{\prime}}$, and (c) the specific and total specific humidities, $\bar{q}$ and $\overline{q_{t}}$, respectively.

This formulation was originally designed to account for the countergradient transport of heat in the clear convective boundary layer. Lock et al. do not apply the nonlocal correction term to $q_{t}$ aiming at making its vertical profiles less well mixed due to the entrainment drying effect. Also, the nonlocal term $\gamma_{q_{t}}$ can sometimes lead to unrealistic humidity profiles that increase with height (Stevens 2000). Note that in mass flux models a nonlocal term appears for any quantity if the vertical velocity distribution is skewed (De Roode et al. 2000; Lappen and Randall 2001). However, in stratocumulus the vertical velocity probability density function is typically rather symmetric such that the nonlocal term will generally be small. In this context it is interesting to explore the representation of stratocumulus with a combined eddy diffusivity/mass flux approach (Soares et al. 2004),

$$
\overline{w^{\prime} \psi^{\prime}}=-K_{\psi} \frac{\partial \bar{\psi}}{\partial z}+M_{c}\left(\psi_{u}-\bar{\psi}\right)
$$

where $M_{c}$ is the convective mass flux, and the subscript $u$ indicates the updraft value of $\psi$.

To stress the importance of the LWP solution, Fig. 6 shows the cloud transmissitivity for broadband solar radiation as a function of the magnitude of the eddy diffusivity. The radiation results were obtained with aid of a broadband delta-Eddington code that is described by Duynkerke et al. (2004). It is obvious that for $c<1$, relatively small changes in the magnitude of the eddy diffusivity can have a dramatic effect on the cloud transmissivity. This strong sensitivity of the LWP on the eddy diffusivity hinders an adequate assessment of the indirect aerosol effect in stratocumulus with GCMs (Johnson 2005).

This note demonstrates that the well mixedness of the boundary layer is controlled by the eddy diffusivity profile applied in a closure model. It can therefore be concluded that a better understanding is needed about the mechanisms that control the degree of well mixedness of stratocumulus cloud layers in nature. In any case, closure models should at least be able to represent near adiabatic stratocumulus cloud layers that were observed during FIRE I by Albrecht et al. (1990) and off the coast of Peru by Bretherton et al. (2004).

Acknowledgments. I thank Jordi Vila and Girmaw Gezahegn from Wageningen University for stimulating discussions on the results of stratocumulus simulations with several boundary layer schemes in MM5. It is a pleasure to thank Andreas Chlond and Frank Müller for their invitation to work on this topic as a visiting scientist at the Max-Planck Institut für Meteorologie in Hamburg, Germany. Finally, I would like to acknowledge my colleagues who participated in the EUROCS stratocumulus intercomparison study and provided the data used in Figs. 3 and 7. This research project was carried out in the framework the Dutch National Research Programme Climate changes Spatial Planning (see online at www.klimaatvoorruimte.nl).

\section{REFERENCES}

Albrecht, B. A., C. W. Fairall, D. W. Thomson, A. B. White, J. B. Snider, and W. H. Schubert, 1990: Surface-based remote sensing of the observed and the adiabatic liquid water content of stratocumulus clouds. Geophys. Res. Lett., 17, 89-92. 
Bougeault, P., and P. Lacarrère, 1989: Parameterization of orography-induced turbulence in a mesobeta-scale model. Mon. Wea. Rev., 117, 1872-1890.

Bretherton, C. S., and Coauthors, 2004: The EPIC 2001 stratocumulus study. Bull. Amer. Meteor. Soc., 85, 967-977.

De Roode, S. R., and P. G. Duynkerke, 1997: Observed Lagrangian transition of stratocumulus into cumulus during ASTEX: Mean state and turbulence structure. J. Atmos. Sci., 54, 2157-2173.

- — - and A. P. Siebesma, 2000: Analogies between massflux and Reynolds-averaged equations. J. Atmos. Sci., 57, $1585-1598$.

, H. J. J. Jonker, P. G. Duynkerke, and B. Stevens, 2004: Counter-gradient fluxes of conserved variables in the clear convective and stratocumulus-topped boundary layer: The role of the entrainment flux. Bound.-Layer Meteor., 112, 179196.

Duynkerke, P. G., and J. Teixeira, 2001: Comparison of the ECMWF reanalysis with FIRE I observations: Diurnal variation of marine stratocumulus. J. Climate, 14, 1466-1478.

- and Coauthors, 2004: Observations and numerical simulations of the diurnal cycle of the EUROCS stratocumulus case. Quart. J. Roy. Meteor. Soc., 130, 3269-3296.

Faloona, I., and Coauthors, 2005: Observations of entrainment in eastern Pacific marine stratocumulus using three conserved scalars. J. Atmos. Sci., 62, 3268-3285.

Holtslag, A. A. M., and B. A. Boville, 1993: Local versus nonlocal boundary layer diffusion in a global climate model. J. Climate, 6, 1825-1842.

Johnson, B. T., 2005: The semidirect aerosol effect: Comparison of a single-column model with large eddy simulation for marine stratocumulus. J. Climate, 18, 119-130.

Jonker, H. J. J., P. G. Duynkerke, and J. W. M. Cuijpers, 1999: Mesoscale fluctuations in scalars generated by boundary layer convection. J. Atmos. Sci., 56, 801-808.

Lappen, C. L., and D. A. Randall, 2001: Toward a unified parameterization of the boundary layer and moist convection. Part I: A new type of mass-flux model. J. Atmos. Sci., 58, 20212036.

Lenderink, G., and A. A. M. Holtslag, 2004: An updated length scale formulation for turbulent mixing in clear and cloudy boundary layers. Quart. J. Roy. Meteor. Soc., 130, 3405-3428. _ , and Coauthors, 2004: The diurnal cycle of shallow cumulus clouds over land: A single column model intercomparison study. Quart. J. Roy. Meteor. Soc., 130, 3339-3364.

Lock, A. P., A. R. Brown, M. R. Bush, G. M. Martin, and R. N. B. Smith, 2000: A new boundary layer mixing scheme. Part I: Scheme description and single-column model tests. Mon. Wea. Rev., 128, 3187-3199.

Louis, J. F., 1979: A parametric model of vertical fluxes in the atmosphere. Bound.-Layer Meteor., 17, 187-202.

Nicholls, S., and J. D. Turton, 1986: An observational study of the structure of stratiform cloud sheets: Part II. Entrainment. Quart. J. Roy. Meteor. Soc., 112, 461-480.

Sánchez, E., and J. Cuxart, 2004: A buoyancy based mixing length proposal for cloudy boundary layers. Quart. J. Roy. Meteor. Soc., 130, 3385-3404.

Soares, P. M. M., P. M. A. Miranda, A. P. Siebesma, and J. Teixeira, 2004: An eddy-diffusivity/mass-flux approach for dry and shallow cumulus convection. Quart. J. Roy. Meteor. Soc., 130, 3365-3384.

Stevens, B., 2000: Quasi-steady analysis of a PBL model with an eddy-diffusivity profile and nonlocal fluxes. Mon. Wea. Rev., 128, 824-836.

_ 2002: Entrainment in stratocumulus-topped mixed layers. Quart. J. Roy. Meteor. Soc., 128, 2663-2690.

_ , and Coauthors, 2005: Evaluation of large-eddy simulations via observations of nocturnal marine stratocumulus. Mon. Wea. Rev., 133, 1443-1462.

Stull, R. B., 1988: An Introduction to Boundary Layer Meteorology. Kluwer Academic Publishers, 666 pp.

Troen, I., and L. Mahrt, 1986: A simple model of the atmospheric boundary layer; sensitivity to surface evaporation. Bound.Layer Meteor., 37, 129-148.

Wyngaard, J. C., and R. A. Brost, 1984: Top-down and bottom-up diffusion of a scalar in the convective boundary layer. $J$. Atmos. Sci., 41, 102-112.

Zhu, P., and Coauthors, 2005: Intercomparison and interpretation of single-column model simulations of a nocturnal stratocumulus-topped marine boundary layer. Mon. Wea. Rev., 133, 2741-2758. 